\title{
Average shape of fluctuations for subdiffusive walks
}

\author{
S. B. Yuste* and L. Acedd \\ Departamento de Física, Universidad de Extremadura, E-06071 Badajoz, Spain
}

(Dated: December 4, 2018)

\begin{abstract}
We study the average shape of fluctuations for subdiffusive processes, i.e., processes with uncorrelated increments but where the waiting time distribution has a broad power-law tail. This shape is obtained analytically by means of a fractional diffusion approach. We find that, in contrast with processes where the waiting time between increments has finite variance, the fluctuation shape is no longer a semicircle: it tends to adopt a table-like form as the subdiffusive character of the process increases. The theoretical predictions are compared with numerical simulation results.
\end{abstract}

PACS numbers: $05.40 .-\mathrm{a}, 05.45 . \mathrm{Tp}, 45.10 . \mathrm{Hj}$

\section{INTRODUCTION}

Complex systems are often described by their lack of a characteristic length or time scale over many orders of magnitude, which gives rise to events whose distribution in sizes is a power law with no characteristic size (fractal behaviour). Examples are everywhere 1]: seismic activity, turbulence, solar flares, Brownian motion, length of rivers and blood vessels,... In particular, a power-law distribution with no characteristic temporal size events appears in the analysis of stock price changes [2, 3], river floods [4], Barkhausen noise [5, 6], glassy systems [7, 8, 9], atomic cooling [10], and fluorescence of quantum dots [1, 12]. In these cases the resulting dynamics is strongly intermittent, with bursts of activity separated by long quiescent intervals.

When these temporal intervals are waiting times $\delta t$ between jumps of size $\delta x$, then the stochastic process $x(t)$ can be seen as the trajectory of a subdiffusive random walker (provided that the variance of $\delta x$ is finite). A typical subdiffusive trajectory is shown in Fig.1. Systems that exhibit anomalous subdiffusion characterized by an anomalous Fick's second law

$$
\left\langle x^{2}(t)\right\rangle \sim \frac{2 K_{\gamma}}{\Gamma(1+\gamma)} t^{\gamma},
$$

where $0<\gamma<1$, are ubiquitous in nature [13, 14]. $\left[K_{\gamma}\right.$ is the (generalized) diffusion constant and $\gamma$ is the anomalous diffusion exponent.] Also, models based on subdiffusive random walkers are useful for understanding complex systems. Two nice examples are the "trap model", proposed to explain aging in disordered systems [7, 8, 9], and the comb model, to understand diffusion phenomena in complex structures such as percolation clusters [15].

Recently [5, 6, 16, 18] the study of the average shape of the fluctuations of stochastic processes $x(t)$ has been considered as a useful tool to gain insight into the system that generates the fluctuation. Thus, it has been argued [18] that the average shape of fluctuations is a

*E-mail: santos@unex.es

†E-mail: acedo@unex.es better tool for discriminating between theories than critical exponents.

In Ref. [16] Baldassarri et al. consider the average shape for a stochastic process of the form $x(t+1)=x(t)+$ $\delta x$, where $\delta x$ is a random variable. Let us denote the average shape of fluctuations of time span $T$ by $\langle x(t)\rangle_{T}$. Baldassarri et al. find that the shape follows the scaling law

$$
\langle x(t)\rangle_{T}=T^{\alpha / 2} f(t / T),
$$

where $\alpha$ is the diffusion exponent and $f$ is a semicircle whenever $\delta x$ follows a distribution with finite variance (Gaussian walks) or a distribution $\lambda(\delta x)$ with a broad power-law tail, $\lambda(\delta x) \sim(\delta x)^{-\mu-1}$ with $0<\mu<2$, so that the variance is infinite (Lévy flights). This result had already been obtained by Fisher for Gaussian walks 19, Sec. 7.1]. The fact that the average shape of fluctuations is a semicircle for both Gaussian walks and Lévy flights is a nicely surprising result that led us to wonder to what extent it might hold for other walks with uncorrelated jumps. In particular, we investigated the average shape of subdiffusive stochastic processes (subdiffusive walks) of the form

$$
x(t+\delta t)=x(t)+\delta x
$$

where $\delta x$ are uncorrelated increments that follow a distribution with finite variance and $\delta t$ is a random vari-

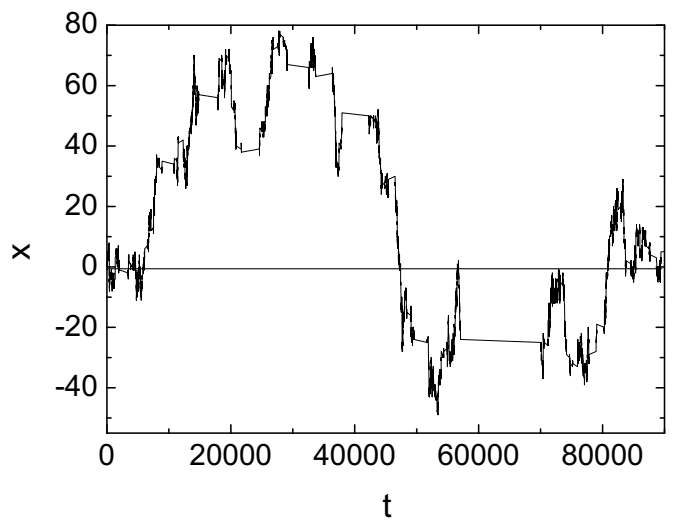

FIG. 1: Subdiffusive trajectory with $\delta x= \pm 1$ and $\gamma=0.9$. 
able whose distribution $\psi(\delta t)$ has a broad power-law tail: $\psi(\delta t) \sim(\delta t)^{-1-\gamma}$ with $0<\gamma<1$. Figure 1 shows the position $x(t)$ for a stochastic process of this kind with $\gamma=0.9$ and with jumps of unit length $(\delta= \pm 1)$ made with equal probabilities.

\section{AVERAGE FORM OF A SUBDIFFUSIVE FLUCTUATION}

The average shape of a fluctuation can be calculated through the expression [16, 17]

$$
\langle x(t)\rangle_{T}=\lim _{x_{0} \rightarrow 0^{+}} \frac{\overline{\int_{0}^{\infty} d x x \Omega\left(x, t \mid\{x(0)\} ; x_{0}, 0, x_{0}, T\right)}}{\int_{0}^{\infty} d x \Omega\left(x, t \mid\{x(0)\} ; x_{0}, 0, x_{0}, T\right)}
$$

where $\Omega\left(x, t \mid\{x(0)\} ; x_{0}, 0, x_{0}, T\right)$ is the probability that the walker with trajectory $\{x(0)\}$ for $t<0$ is at $x$ at time $t$ provided that he was at $x_{0}>0$ at time 0 and at $x_{0}$ at time $T$ without ever touching the axis at $x=0$ in the time interval $(0, T)$. The upper line in Eq. (4) means average over all the trajectories $\{x(0)\}$ that reach $x_{0}$ at time $t=0$. Let $F\left(x, t \mid\left\{x\left(t^{\prime}\right)\right\} ; x^{\prime}, t^{\prime}\right)$ be the probability that the walker with trajectory $\left\{x\left(t^{\prime}\right)\right\}$ for $t<t^{\prime}$ and who was at $x^{\prime}>0$ at time $t^{\prime}$ reaches $x>0$ at time $t$ without ever touching the axis at $x=0$; let $\Omega\left(x, t \mid x_{0}, 0, x_{0}, T\right)$ be the probability that the walker is at $x$ at time $t$ provided that he was at $x_{0}>0$ at time 0 and at $x_{0}$ at time $T$ without ever touching the axis at $x=0$ in the time interval $(0, T)$; and let $F\left(x, t \mid x^{\prime}, t^{\prime}\right)$ be the probability that the walker who was at $x^{\prime}$ at time $t^{\prime}$ reaches $x>0$ at time $t$ without ever touching the axis at $x=0$. For walks without memory (Markovian walks) $\Omega\left(x, t \mid\{x(0)\} ; x_{0}, 0, x_{0}, T\right)=\Omega\left(x, t \mid x_{0}, 0, x_{0}, T\right)$, $F\left(x, t \mid\left\{x\left(t^{\prime}\right)\right\} ; x^{\prime}, t^{\prime}\right)=F\left(x, t \mid x^{\prime}, t^{\prime}\right)$, and one can write $\Omega\left(x, t \mid x_{0}, 0, x_{0}, T\right)$ as $F\left(x, t \mid x_{0}, 0\right) F\left(x, T-t \mid x_{0}, 0\right)$, so that Eq. (4) becomes [16, 17]

$$
\langle x(t)\rangle_{T}=\lim _{x_{0} \rightarrow 0^{+}} \frac{\int_{0}^{\infty} d x x F\left(x, t \mid x_{0}, 0\right) F\left(x, T-t \mid x_{0}, 0\right)}{\int_{0}^{\infty} d x F\left(x, t \mid x_{0}, 0\right) F\left(x, T-t \mid x_{0}, 0\right)} .
$$

This equation is not exact for subdiffusve walks because they are not Markovian. However, for subdiffusive walks, the memory, i.e., the effect of the fact that at $t^{\prime}$ the particle was at $x^{\prime}$ on the probability that the particle at time $t>t^{\prime}$ is at $x$, decays as $\left(t-t^{\prime}\right)^{-\gamma} / \Gamma(1-\gamma)$ 13]. This implies that the approximation of $F\left(x, t \mid\left\{x\left(t^{\prime}\right)\right\} ; x^{\prime}, t^{\prime}\right)$ by $F\left(x, t \mid x^{\prime}, t^{\prime}\right)$, and, consequently, the accuracy of Eq. (5) for subdiffusive walks, improves when $t-t^{\prime}$ increases and $\gamma$ is close to 1 .

The probability $F\left(x, t \mid x_{0}, 0\right)$ can be calculated by means of the method of images $F\left(x, t \mid x_{0}, 0\right)=P(x-$ $\left.x_{0}, t\right)-P\left(-x-x_{0}, t\right)[20,21], P\left(x-x_{0}, t\right)$ being the probability density that the free process (without boundary conditions) that at time $t \leq 0$ was at $x_{0}$ is at $x$ at time $t$. For subdiffusive processes, and for $t^{-\gamma} / \Gamma(1-\gamma) \ll 1$ 22], $P(x, t)$ can be written in terms of Fox's $H$ function as [13]:

$$
P(x, t)=\frac{1}{\sqrt{4 K_{\gamma} t^{\gamma}}} H_{11}^{10}\left[\frac{|x|}{\sqrt{K_{\gamma} t^{\gamma}}} \mid \begin{array}{l}
(1-\gamma / 2, \gamma / 2) \\
(0,1)
\end{array}\right] .
$$

Taking into account that the Laplace transform of $P(x, t)$ is

$$
P(x, u)=\frac{u^{\gamma / 2-1}}{\sqrt{4 K_{\gamma}}} \exp \left(-\sqrt{u^{\gamma} / K_{\gamma}}|x|\right)
$$

one finds for $x \geq 0$

$$
\begin{aligned}
F\left(x, u \mid x_{0}, 0\right) & =\frac{u^{\gamma / 2-1}}{\sqrt{4 K_{\gamma}}}\left\{e^{-a\left(x_{0}-x\right)}\left[\Theta(x)-\Theta\left(x-x_{0}\right)\right]\right. \\
& \left.+e^{-a\left(x-x_{0}\right)} \Theta\left(x-x_{0}\right)-e^{-a\left(x+x_{0}\right)}\right\}, \quad(8
\end{aligned}
$$

where $a \equiv \sqrt{u^{\gamma} / K_{\gamma}}$ and $\Theta(x)$ is the Heaviside step function. As we are interested in the limit $x_{0} \rightarrow 0$ with $x>x_{0}$, we expand the term inside the bracket in powers of $x_{0}$ and get

$$
F\left(x, u \mid x_{0} \rightarrow 0,0\right)=x_{0} \frac{u^{\gamma-1}}{K_{\gamma}} e^{-\sqrt{u^{\gamma} / K_{\gamma}} x}
$$

which implies

$$
F\left(x, t \mid x_{0} \rightarrow 0,0\right)=\frac{x_{0}}{K_{\gamma} t^{\gamma}} H_{11}^{10}\left[\begin{array}{l|l}
\frac{x}{\sqrt{K_{\gamma} t^{\gamma}}} & \begin{array}{l}
(1-\gamma, \gamma / 2) \\
(0,1)
\end{array}
\end{array}\right] .
$$

Inserting this expression into Eq. (5) and carrying out the integrations 23] one finds that the average shape of a subdiffusive stochastic process (subdiffusive random walk) is given by

$$
\langle x(t)\rangle_{T}=T^{\gamma / 2} f_{\gamma}(t / T)=\sqrt{K_{\gamma} T^{\gamma}} g_{\gamma}(t / T)
$$

where

$$
g_{\gamma}(t / T)=\frac{\left(\frac{t}{T}\right)^{\gamma / 2} H_{22}^{11}\left[\left(\frac{t / T}{1-t / T}\right)^{\gamma / 2} \mid \begin{array}{l}
(-1,1),\left(1-\gamma, \frac{\gamma}{2}\right) \\
(0,1),\left(0, \frac{\gamma}{2}\right)
\end{array}\right]}{H_{22}^{11}\left[\begin{array}{l|l}
\left(\frac{t / T}{1-t / T}\right)^{\gamma / 2} & \left.\begin{array}{l}
(0,1),\left(1-\gamma, \frac{\gamma}{2}\right) \\
(0,1),\left(\frac{\gamma}{2}, \frac{\gamma}{2}\right)
\end{array}\right]
\end{array} .\right.}
$$

In Fig. 2 we plot the (normalized) average shape of fluctuations for several classes of subdiffusive processes. We see that the shape tends to a table-like form as $\gamma$ decreases, i.e., as the subdiffusive character of the process increases.

Of course, for $\gamma \rightarrow 1$ one recovers the Gaussian result $f_{1}(t / T)=\sqrt{16 D / \pi} \sqrt{t / T(1-t / T)}[16,19]$ because the upper and lower Fox's $H$ functions in Eq. (12) become $2 z / \pi\left(1+z^{2}\right)^{2}$ and $z / 2 \sqrt{\pi}\left(1+z^{2}\right)^{3 / 2}$, respectively.

The area $N(T)$ of the average fluctuation of duration $T$ is given by

$$
N(T)=\int_{0}^{1} d s\langle x(s T)\rangle_{T}
$$




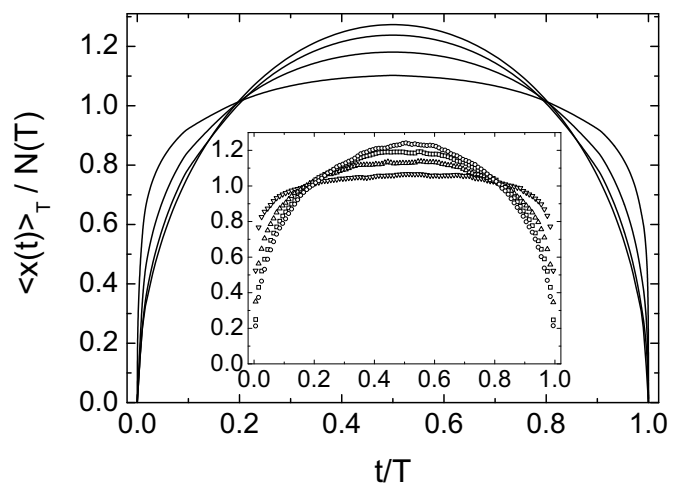

FIG. 2: Normalized average fluctuation for subdiffusive processes for several values of $\gamma$. The shape is normalized so that its area is 1 , i.e., we plot $\langle x(t)\rangle_{T} / N(T)$. The lines are the theoretical result for (at $t / T=1 / 2$ and from top to bottom) $\gamma=1,0.9,0.75,0.5$. Inset: simulation results for these same values of $\gamma$.

TABLE I: The coefficient $n_{\gamma}=\int_{0}^{1} d s g_{\gamma}(s)$ calculated by numerical integration.

\begin{tabular}{ccccccccc}
\hline \hline$\gamma$ & $1 / 4$ & $1 / 3$ & $1 / 2$ & $2 / 3$ & $3 / 4$ & $4 / 5$ & $9 / 10$ & 1 \\
$n_{\gamma}$ & 0.493 & 0.501 & 0.537 & 0.612 & 0.668 & 0.708 & 0.798 & $\sqrt{\pi} / 2$ \\
\hline \hline
\end{tabular}

where $s=t / T$. From Eq. (11) one finds

$$
N(T)=n_{\gamma} \sqrt{K_{\gamma} T^{\gamma}}
$$

where $n_{\gamma}=\int_{0}^{1} d s g_{\gamma}(s)$. Then, the normalized average fluctuation is given by

$$
\frac{\langle x(t)\rangle_{T}}{N(T)}=\frac{g_{\gamma}(t / T)}{n_{\gamma}} .
$$

We have not been able to calculate $n_{\gamma}$ analytically. In Table 【 we give some values evaluated numerically.

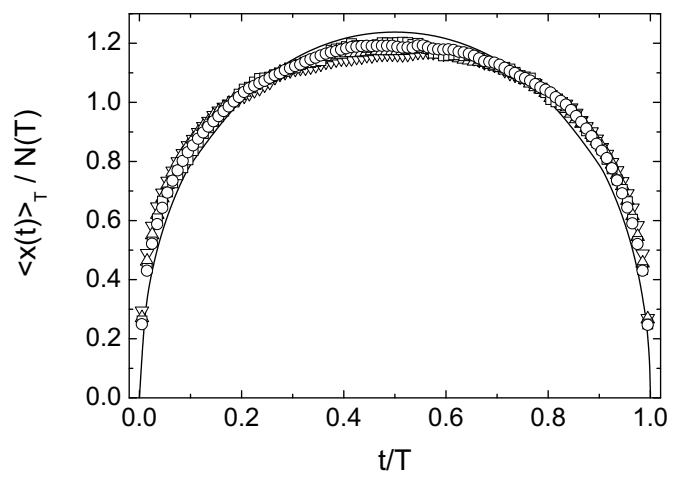

FIG. 3: Normalized average fluctuation for the subdiffusive process with $\gamma=0.9$. The line is the theoretical result and the symbols are simulation results for $T=10^{4}, 10^{5}, 10^{6}, 10^{7}, 10^{8}$.

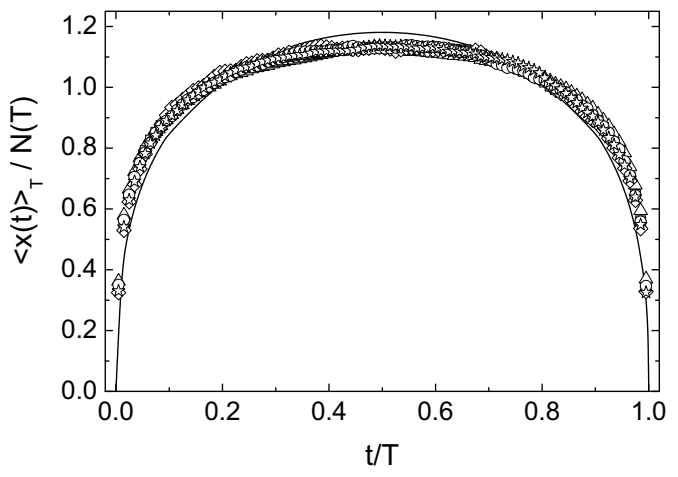

FIG. 4: Normalized average fluctuation for the subdiffusive process with $\gamma=0.75$. The line is the theoretical result and the symbols are simulation results for $T=$ $10^{4}, 10^{5}, 10^{6}, 10^{7}, 10^{8}$.

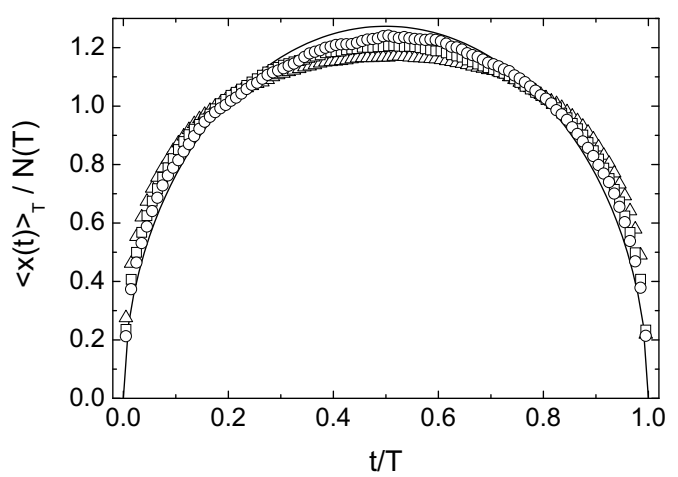

FIG. 5: Normalized average fluctuation for a process with $\gamma=1$. The line is the semicircular form theoretical result and the symbols are simulation results for $T=10^{3}$ (triangles), $10^{5}$ (squares), and $10^{7}$ (circles).

\section{SIMULATION}

We carried out simulations of the fluctuations of the stochastic process (3) where $\delta x$ takes the values +1 or -1 with equal probabilities and where the waiting time $\delta t$ between jumps follows the Pareto distribution $\psi(t)=$ $\gamma /(1+t)^{1+\gamma}$. In this case, the diffusion constant $K_{\gamma}$ is given by 13] $K_{\gamma}=1 /[2 \Gamma(1-\gamma)]$. The simulation results follow the pattern found in the precedent section: the fluctuation shape tends toward a table-like form as $\gamma$ decreases (see inset in Fig. 2).

In Figs. 3] and 4 we compare the theoretical predictions and the simulation results for $\gamma=0.9$ and $\gamma=0.75$, respectively. The agreement is reasonable. We attribute the differences to, first, the approximate nature of Eq. (5) for non-Markovian processes and, second, to a slow convergence that requires longer $T$ to set in. This can be clearly appreciated in Fig. 5 where one sees that the wellestablished theoretical semicircular shape is approached, although very gradually, as $T$ increases . It is even more gradual for smaller $\gamma$. We have not explored larger values for $T$ because of the excessive computer time required. 


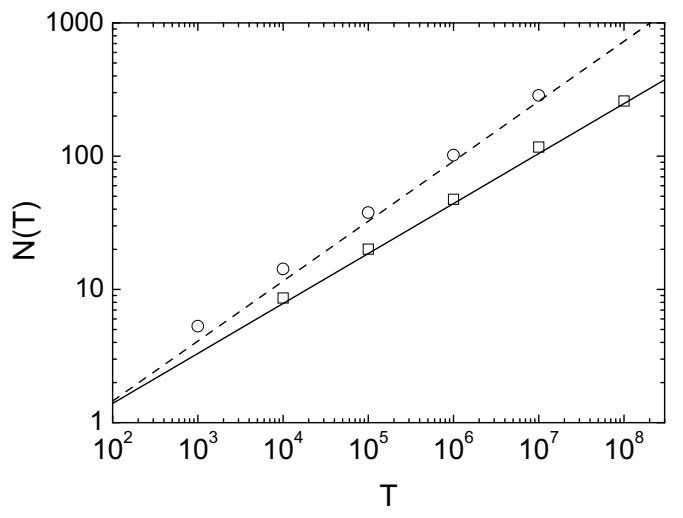

FIG. 6: Area $N(T)$ of the fluctuation for $\gamma=0.75$ (circles) and $\gamma=0.9$ (squares). The lines are the corresponding theoretical prediction; see Eq. (14).

In the simulations we also calculated the area $N(T)$ of the fluctuation. In Fig. 6 we plot $N(T)$ for several values of $T$ and for two values of $\gamma$. The agreement between simulation and theory is reasonable again.

\section{CONCLUSIONS}

We have analyzed the average shape of the fluctuations of time series generated by the stochastic process (3) with a power-law waiting time distribution $\psi(t) \sim t^{-1-\gamma}$ where $0<\gamma<1$ (subdiffusive walks). Although the spatial increments $\delta x$ in this stochastic process are uncorrelated, we find that, in contrast with Gaussian walks and Lévy flights, the average shape of a fluctuation is no longer a semicircle: its form becomes flatter at the top and steeper at the extremes as the subdiffusive character of the process increases (i.e., as $\gamma$ decreases).

Some possible sequels of the present work are obvious: one could consider the effect of correlated increments $\delta x$ (as done by Baldassarri et al. 16]) or investigate the average shape of a fluctuation for Lévy walks (in which $|\delta x|$ and $\delta t$ are proportional and follow a broad power-law tail). However, for these cases, the task of getting analytical results, even approximate, such as those reported in the present paper certainly looks formidable.

\section{Acknowledgments}

We are grateful to Francesca Colaiori, Andrea Baldassarri and Claudio Castellano for their useful remarks and for sharing with us Ref. [17] prior to publication. This work was supported by the Ministerio de Ciencia y Tecnología (Spain) through Grant No. FIS200401399 and by the European Community's Human Potential Programme under contract HPRN-CT-2002-00307, DYGLAGEMEM.
[1] H. Takayasu, Fractals in the Physical Sciences (Wiley, Chichester, 1992); Fractals in Science, edited by A. Bunde and S. Havlin (Springer-Verlag, Berlin, 1994); M. Schroeder, Fractals, Chaos, Power Laws: Minutes from an Infinity Paradise (Freeman, New York, 1991).

[2] J.-P. Bouchaud, e-print cond-mat/0008103 http://xxx.lanl.gov

[3] P. Bak, M. Paczuski and M. Shubik, Physica A 246, 430 (1997).

[4] J. Feder, Fractals (Plenum, New York, 1988).

[5] M. C. Kuntz and J. P. Sethna, Phys. Rev. B 62, 11699 (2000).

[6] D. Spasojević, S. Bukvić, S. Milošević and H. E. Stanley, Phys. Rev. E 54, 2531 (1996).

[7] J.-P. Bouchaud, J. Phys. I 2, 1705 (1992).

[8] G. Ben Arous, A. Bovier and V. Gayrard, Phys. Rev. Lett. 88, 087201 (2002).

[9] E. M. Bertin and J.-P. Bouchaud, Phys. Rev. E 67, 026128 (2003).

[10] B. Saubaméa, M. Leduc and C. Cohen-Tannoudji, Phys. Rev. Lett. 83, 3796 (1999).

[11] K. T. Shimizu, R. G. Neuhauser, C. A. Leatherdale, S. A. Empedocles, W. K. Woo and M. G. Bawendi, Phys. Rev. B 63, 205316 (2001).
[12] X. Brokmann, J.-P. Hermier, G. Messin, P. Desbiolles, J.P. Bouchaud and M. Dahan, e-print cond-mat/0211171 http://xxx.lanl.gov

[13] R. Metzler and J. Klafter, Phys. Rep. 339, 1 (2000).

[14] J.-P. Bouchaud and A. Georges, Phys. Rep. 195, 127 (1990).

[15] S. Havlin and D. Ben-Avraham, Adv. Phys. 36, 695 (1987).

[16] A. Baldassarri, F. Colaiori and C. Castellano, Phys. Rev. Lett. 90, 060601 (2003).

[17] F. Colaiori , A. Baldassarri and C. Castellano, e-print cond-mat/0402285 http://xxx.lanl.gov

[18] J. P. Sethna, K. A. Dahmen and C. R. Myers, Nature 410, 242 (2001).

[19] M. Fisher, J. Stat. Phys. 34, 667 (1984).

[20] S. Redner, A Guide to First-passage Processes (Cambridge University Press, Cambridge, 2001).

[21] R. Metzler and J. Klafter, Physica A 278, 107 (2000).

[22] E. Barkai, R. Metzler and J. Klafter, Phys. Rev. E 61, 132 (2000).

[23] A. M. Mathai and R. K. Saxena, The H-function with Applications in Statistics and Other Disciplines (Wiley, New York, 1978). 